\title{
Belgeo
}

Revue belge de géographie

1 | 2008

Aspects of the history of the Belgian geography and cartography

\section{Vellut Jean-Luc (dir.), Villes d'Afrique. Explorations en histoire urbaine}

Cahiers Africains, $\mathrm{n}^{\circ} 73$, Africa Tervuren, FNRS, Chaire d'études africaines de l'UCL, Editions l'Harmattan, 2007, 237 p.

\section{Henri Nicolaï}

\section{(2) OpenEdition}

Édition électronique

URL : http://journals.openedition.org/belgeo/10231

DOI : 10.4000/belgeo.10231

ISSN : 2294-9135

Éditeur :

National Committee of Geography of Belgium, Société Royale Belge de Géographie

Édition imprimée

Date de publication : 31 mars 2008

Pagination : 147-149

ISSN : 1377-2368

Référence électronique

Henri Nicolaï, «Vellut Jean-Luc (dir.), Villes d'Afrique. Explorations en histoire urbaine », Belgeo [En ligne], 1 | 2008, mis en ligne le 19 octobre 2013, consulté le 22 septembre 2020. URL : http:// journals.openedition.org/belgeo/10231; DOI : https://doi.org/10.4000/belgeo.10231

Ce document a été généré automatiquement le 22 septembre 2020.

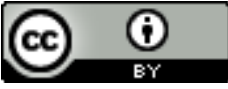

Belgeo est mis à disposition selon les termes de la licence Creative Commons Attribution 4.0 International. 


\section{Vellut Jean-Luc (dir.), Villes d'Afrique. Explorations en histoire urbaine}

Cahiers Africains, $n^{\circ} 73$, Africa Tervuren, FNRS, Chaire d'études africaines de l'UCL, Editions l'Harmattan, 2007, 237 p.

\section{Henri Nicolaï}

\section{RÉFÉRENCE}

Vellut Jean-Luc (dir.), Villes d'Afrique. Explorations en histoire urbaine, Cahiers Africains, $\mathrm{n}^{\circ} 73$, Africa Tervuren, FNRS, Chaire d'études africaines de l'UCL, Editions l'Harmattan, 2007, 237 p.

1 Pour l'historien des villes et pour le géographe curieux de voir comment naît et se développe un centre urbain, l'Afrique tropicale est fascinante. C'est assurément la partie du monde qui, au XX ${ }^{\mathrm{e}}$ siècle, a vu apparaître et croître le plus grand nombre de villes, souvent nées de rien sinon d'un germe antérieur à peine visible. Sans doute est-il excessif d'attribuer à toute ville d'Afrique Noire une origine exclusivement coloniale. De toute façon, le développement a échappé très vite au contrôle colonial même avant l'accession à l'indépendance politique. Il n'en reste pas moins qu'à l'exception de quelques noyaux traditionnels intérieurs, restés souvent d'ailleurs assez petits, l'essentiel des villes actuelles est le résultat d'un contact direct ou indirect avec le monde extérieur, c'est-à-dire surtout, dans le cas de l'Afrique centrale, avec le monde occidental. On peut gloser à l'infini sur ce thème et chercher à mesurer l'apport respectif des facteurs endogènes et des facteurs exogènes. Le fait fondamental est qu'une fois amorcée, la ville africaine a connu son évolution propre.

Ce livre collectif, que vient de diriger Jean-Luc Vellut, doit retenir au plus haut point l'attention des géographes. L'histoire des villes africaines y est abordée de façon multidisciplinaire ou plus exactement chaque contribution représente une approche particulière. Certes l'approche géographique proprement dite n'y est pas présente sinon par une contribution à caractère surtout démographique sur la transformation 
du réseau urbain de la R.D.C. (par Léon de Saint Moulin) mais plusieurs contributions révéleront au géographe des aspects intéressants et parfois surprenants qui marquent ou qui expliquent le paysage des villes actuelles et dont il doit se préoccuper.

Dans une contribution générale sur les approches critiques de la cité africaine, Zynep Ç elik, une historienne de l'architecture (Institut de technologie du New Jersey) montre qu'on est passé, dans l'histoire urbaine en général, et dans celle de l'Afrique Noire en particulier, du moins dans l'université états-unienne, d'une phase où prédominait le schématique à une phase où prédomine le concret (microhistoire, enquête à petite échelle) et, depuis les années 1980, à une "reconceptualisation de l'espace urbain en termes de relations sociales" et à la "compréhension des significations prises par les formes". Une idée aussi qui prédomine actuellement est qu'il n'y a pas "une" Cité africaine mais "de multiples villes, chacune avec ses conditions spécifiques, son histoire, sa vie et ses formes".

4 Nous avons dit plus haut qu'un des intérêts de ce livre était la diversité des approches. Nous retiendrons celles auxquelles les géographes sont le plus sensibles. Signalons simplement, parmi celles qui les concernent moins, les contributions suivantes. D'abord un chapitre sur l'architecture urbaine catholique à Kinshasa, par Pamphile Mabiala Mantuba-Ngoma, qui nous montre le souci, à partir de 1958, de construire des églises "tropicales" (!), la "tropicalisation" étant considérée comme une "indigénisation" du bâtiment (évolution des temps: toutes les églises de Kinshasa sont aujourd'hui entourées d'une clôture avec une grille métallique d'entrée). Ensuite un article de Françoise Hiraux sur la représentation des villes et des valeurs urbaines dans la colonie belge, étudiée à partir d'un collection de photographies issues de l'agence gouvernementale Congo Presse, c'est-à-dire d'un organisme de propagande qui exprime évidemment la perception des autorités coloniales et qui veut surtout montrer comment, avec la colonisation, la population congolaise tend de plus en plus à assimiler les valeurs et les comportements de la société occidentale. Une communication, par Donatien Dibwe dia Mwembu décrit une opération de sensibilisation de la population de Lubumbashi à la mémoire de sa ville et notamment de son passé industriel, à travers plusieurs expositions organisées par le département d'histoire de l'université au musée national de Lubumbashi.

5 Un article sur la côte du Tanganyika (de Katrin Bromber) montre que, déjà dans les années 1920, la presse locale se demandait si le buibui, ce long voile porté par les femmes citadines était un vêtement traditionnel ou un signe d'appartenance religieuse. N'était-ce pas aussi pour les citadines, le moyen d'exprimer leur appartenance à la civilisation de la côte, c'est-à-dire à une forme de civilisation jugée supérieure par opposition à celle des femmes venues des campagnes intérieures.

6 Il ne faut pas négliger le rôle de certains groupes dans la formation de la citoyenneté urbaine. Les anciens combattants des deux guerres mondiales se sont installés souvent dans des villes après leur démobilisation. Leur cas est étudié à Bobo Dioulasso (Burkina Faso) par Brigitte Reinwald. On remarquera que si, grâce à leurs pensions, ils ont pu former un groupe un peu plus aisé que les immigrants ruraux, ils ont très peu investi dans les activités urbaines sinon dans la création de cabarets et d'espaces de loisirs où leur rôle a été important.

7 Parmi les contributions qui retiendront plus spécifiquement l'attention des géographes, signalons tout d'abord un article de Rik Ceysens, intitulé "Urbanisme et vision de la ville dans les royaumes de la savane" qui décrit les centres des royaumes kanyok, ruund 
(lunda) et luba dans les savanes du Kasai oriental et du Katanga occidental. Aujourd'hui par exemple une série d'îlots forestiers englobant les tombes des rois kanyok occupent le lieu où se trouvait le sérail du roi vivant. Il $\mathrm{y}$ avait donc des capitales politiques aménagées généralement sur une crête, selon un modèle comportant un plan orienté et une organisation en quartiers. Au centre, le bâtiment prestigieux du roi ; en aval, la cuisine pour le roi et ses invités, en amont une place semi-publique pour les audiences et plus en amont encore, la résidence de la reine-mère où se trouvait l'entrée publique. Des quartiers homogènes (épouses fécondes, épouses stériles, notables, artisans, guerriers) s'organisaient tout autour, reproduisant l'image du pays. C'est à côté de cette capitale que venaient camper les commerçants étrangers, les hommes fuyant d'autres territoires et où se sont installés, par la suite, les représentants de l'autorité coloniale. Il est fâcheux que ce thème très intéressant soit traité de façon fort confuse et que l'auteur, dans sa première ligne, situe le pays des Kanyok à des altitudes andines ou tibétaines ( $4000 \mathrm{~m}$ ! sans doute s'agit-il de pieds et non de mètres et encore !).

Un article de Léon de Saint Moulin retrace la croissance démographique de Kinshasa depuis 1920 (25000 habitants en 1920, un peu plus de 7 millions en 2004) et analyse une image satellitaire de 1995. Il comporte ensuite une vue d'ensemble du réseau urbain (villes et cités) de la République démocratique du Congo en 2004 avec des tableaux donnant la population de chaque localité urbaine en 1958, 1970, 1975, 1984 et 2004 et un classement par taille et taux de croissance (25 villes ou centres de plus de 100000 habitants, 19 de 50000 à 100000 ; avec 135 centres plus petits, cela fait $34,5 \%$ de la population du pays).

9 Des maisons de style "afro-brésilien" marquent le paysage urbain de certaines villes côtières du Bénin actuel et du pays yoruba au Nigéria. Une intéressante contribution de Brigitte Kowalski étudie leur diffusion depuis les cités de la Côte des Esclaves jusqu'au centre des pays egba (Abeokuta) et egbado. Il s'agit de ces maisons construites par des Africains revenus du Brésil à partir du début du XIX ${ }^{e}$ siècle, esclaves affranchis, qui ont joué d'abord un grand rôle dans la traite finissante puis dans les nouvelles activités qui lui ont succédé comme le commerce de l'huile de palme. Ces maisons à étage, aux murs de briques, aux motifs colorés, de style portugais et même ces églises et ces mosquées rappelant le baroque brésilien ont été construites parfois par des maçons qui avaient appris leur métier à Bahia. Il faut citer aussi le rôle des Saro, revenus au Sierra Leone, esclaves affranchis ou esclaves débarqués à Freetown après l'arraisonnement de navires négriers par la flotte anglaise.

La conception urbanistique de la capitale du Congo belge se réfère pour de nombreux points aux caractéristiques de certains quartiers de Bruxelles. John Lagae, à qui on doit d'intéressantes études sur l'architecture des villes coloniales africaines du XX siècle, pose la question : Léopoldville et Bruxelles ne sont-elles pas des villes miroirs ? Dès les années 1920, des plans ont été dressés pour le quartier où devaient s'installer l'administration générale de la colonie et les résidences des hauts fonctionnaires belges, c'est-à-dire le quartier de Kalina (actuellement Ngombe) en bordure du fleuve. Le premier modèle a été le quartier bruxellois du Parc où se côtoyaient pouvoir exécutif, direction des milieux d'affaires (y compris les affaires coloniales) et pouvoir législatif. Par la suite, c'est plutôt le Mont des Arts qui a servi de référence. En tout cas les péripéties de la construction d'une résidence pour le gouverneur général, qui aurait été à la hauteur de sa fonction, sont très instructives. On imagina d'abord quelque chose qui ressemblait au bâtiment du Musée de Tervuren et au Palais Royal de 
Bruxelles, puis un palais de style mauresque avec une tour minaret, qu'on considérait comme adaptée à un monde tropical mais dont l'aspect était en contradiction avec les mythes fondateurs du Congo. Le bâtiment qui fut enfin construit dans les années 1950 n'était d'ailleurs pas achevé en 1960 et fut utilisé comme siège du parlement du nouvel Etat. En fait, les autorités belges de Bruxelles ont toujours été réticentes devant une construction qui aurait pu faire croire que le gouverneur général disposait d'un pouvoir et d'une autonomie que l'on n'a jamais voulu lui accorder.

11 Deux contributions concernent directement ou indirectement la question de la ségrégation.

12 A Douala (article de Andreas Eckert), la ségrégation a abouti à trois parties distinctes, la ville européenne, la ville duala, la ville "africaine", c'est-à-dire celle des immigrants. Avant l'occupation allemande, les pêcheurs duala avaient organisé un port qui s'occupait aussi de commerce (esclaves, huile de palme). Il y avait là une aristocratie locale (quatre grands villages avec chacun un "roi") qui a cherché à maintenir sa place dans la ville aménagée par l'administration coloniale allemande selon les principes d'une ségrégation raciale s'appuyant sur des arguments sanitaires. Les Duala se sont opposés ainsi aux mesures d'expropriation les écartant des rives du fleuve. S'ils n'y ont pas réussi, ils ont obtenu cependant de pouvoir s'installer dans des quartiers où leurs résidences rivalisent avec celles de la cité européenne. Le cas de Douala montre bien que cette ségrégation tripartite est le résultat des "stratégies contradictoires et mêlées de tous les intéressés, Africains et Européens", et non de la seule action du pouvoir colonial.

La ville sud-africaine de Pietermaritzburg (KwaZulu-Natal), fondée en 1838 par des Voortrekkers hollandais venus de la région du Cap, portait les marques de la ségrégation raciale, d'abord à l'égard des Indiens puis des Africains, bien avant qu'on y appliquât la politique de l'apartheid (contribution de Philippe Denis). Malgré l'évolution de la législation, les différences entre quartiers blancs, quartiers indiens, quartiers noirs et quartiers colorés sont aujourd'hui encore très visibles. Le centre de la ville, autrefois blanc, s'est prolétarisé. Dans quelques cas, la ségrégation sociale s'est ajoutée aussi à la ségrégation ethnique ou l'a remplacée.

La diversité de toutes ces contributions, dont nous n'avons évoqué que quelques aspects, fait de cet ouvrage, un livre particulièrement utile aux géographes qui s'occupent du monde urbain africain. 